\title{
Desenvolvimento de um revolvedor mecânico de café
}

\section{Development of a mechanical homogenizer coffee}

\author{
Raphael Magalhães Gomes Moreira ${ }^{1 *}$; Mauri Martins Teixeira ${ }^{2}$; \\ Haroldo Carlos Fernandes²; Paulo Roberto Cecon ${ }^{3}$; Luciano José Minette ${ }^{3}$
}

\section{Resumo}

A cafeicultura demanda investimentos para o desenvolvimento de máquinas que viabilizem os processos pós-colheita, dando celeridade e melhorando as condições de trabalho. A utilização de secadores de camada estacionária resulta em bons resultados quanto à secagem e qualidade do produto, mas necessitam de revolvimento constante para homogeneizar a massa de grãos. A escassez de mão de obra associada à necessidade de revolvimento constante e as pesadas condições de trabalho obrigam a mecanização desta etapa. O projeto de uma máquina agrícola é considerado de grande complexidade, preocupando-se com as interações entre o operador, a máquina e o ambiente. Ao projetar uma máquina, as experiências e o domínio sobre os diversos pontos de vista tem que ser interpretados de forma clara. Com o aumento da competitividade, diversas empresas de máquinas e implementos agrícolas tem buscado os centros de pesquisa, pois em geral não utilizam dos procedimentos sistemáticos durante o projeto, o que pode resultar em falhas de operação. Objetivou-se com a realização deste trabalho projetar e construir um protótipo revolvedor semimecanizadopara realizar a mistura dos grãos de café. O protótipo revolvedor com o princípio de rosca helicoidal se mostrou capaz de realizar a movimentação dos grãos com eficiência.

Palavras-chave: Projeto de máquinas, secagem, máquinas para café

\begin{abstract}
The crop Coffee demands investments to the machines development, which it enables the processes ofpost-harvesting, becoming them faster, meanwhile improving the working. The use of stationary layer dryers are responsible for good results at drying and product quality, but it requires a constant revolving, in order to homogenize the grain mass and improve the air distribution inside the drying chamber. The shortage of workmanship, associated to the need of constant revolving and the heavy working conditions, it becomes it indispensable to mechanize, in some way, this step. The agricultural machine design is considered of great complexity, regarding it must be concerned with the interactions among the operator, machine and environment. When designing a machine, the experience and the dominion of several standpoints have to be interpreted clearly. With the increasing competitiveness on the consumer market and agricultural machines, several companies have joined the research centers, because in general, do not make use of systematic procedures during the project, which can result in failures during operation. This article aimed to design and build a semi-mechanized revolving prototype used to mix the coffee beans. The revolving prototype with the helical screw principles proved to be able to performing the grains transport efficiently.
\end{abstract}

Key words: Machine design, drying process, coffee machines

\footnotetext{
${ }^{1}$ Eng $^{\circ}$ Agrícola e Ambiental, Prof. do Instituto Federal do Espírito Santo, Campus Itapina, Discente de Doutorado, Programa de Pós-Graduação em Eng ${ }^{\circ}$ Agrícola, Dept ${ }^{\circ}$ de Engenharia Agrícola, DEA, Universidade Federal de Viçosa, UFV, Viçosa, MG. E-mail: raphael.moreira@ifes.edu.br

${ }^{2}$ Profs. da UFV/DEA, Viçosa, MG. E-mail: mauri@ufv.br; haroldo@ufv.br

${ }^{3}$ Profs. da UFV, Viçosa, MG. E-mail: cecon@ufv.br; minette@ufv.br

* Autor para correspondência
} 
O Estado de Minas Gerais participa com 67,6\% da produção de café arábica do país, tendo o Brasil como o maior produtor de café e o segundo mercado consumidor mundial.O principal objetivo do setor cafeeiro brasileiro é recuperar as vendas no exterior, e para isso, se faz necessário aumentar a qualidade do café buscando por alternativas nos sistemas produtivos e de pós-colheita (SILVA et al., 2011; SAATH et al., 2010).

A secagem artificial é uma das principais operações de pós-colheita por representar grande parte do custo operacional da produção, por agilizar o processo de retirada de água, reduzir a utilização de mão-de-obra e permitir o armazenamento dos produtos, sem que estes sofram perdas de qualidade (RIGUEIRA et al., 2007; CORADI; BOREM; OLIVEIRA, 2008; AMPESSAN et al., 2010).

Dentre os diversos secadores artificiais que sãomuito utilizados paradiminuir o tempo de secagem em situações de condições climáticas adversas (CHAGAS; TEIXEIRA; PRAT, 2006), destacam-se os secadores de camada estacionária. Estes demandam revolvimento da massa de grãos em intervalos regularescom o uso de enxadas ou pás, o que torna essa operação muito árdua.

A escassez e o aumento do custo da mãodeobra, somadas às precárias condições de trabalho, refletem na crescente necessidade de se mecanizar todas as operações necessárias à cafeicultura, sendo que esta necessidade não tem a função de gerar desemprego, mas sim amenizar os serviços pesados e aumentar a capacidade de produção do trabalhador, reduzindo o custo da colheita e da pós-colheita.Ascaracterísticas operacionais e construtivas dasmáquinasagrícolas vendidas no exterior tem que ser adequadaspara o mercado brasileiro, já que a manutenção dessas propriedades pode resultar em perda de qualidade das máquinas, elevação dos custos de aquisição e problemas ergonômicos (MINETTE et al., 2007, 2008).

Com objetivo de simular o funcionamento de protótipos, verificar as condições de funcionamento e durabilidade das peças que fazem parte da constituição das máquinas,podem ser utilizados programas de computador com base no sistema CAD (Computer Aided Design) (MAGALHÃES et al., 2006).

Os programas $\mathrm{CAD}$ auxiliam no projeto, que é umametodologia inovadora e altamente interativa. É também um processo de tomada de decisões, que podem ser feitas com poucas informações. O processo de projeto começa com o reconhecimento de uma necessidade e de uma decisão envolvendo algo ou planos para satisfazer tal necessidade, (SHIGLEY; MISCHKE; BUDYNAS, 2004). A falta de aplicação dos conhecimentos de engenharia e de metodologia de projeto pode causar erros do mesmo, resultando em falhas durante a operação e acidentes dos operadores (SANTOS et al., 2008). Com o objetivo de nortear as fábricas é preciso realizar testes e avaliações buscando melhorias das máquinas (ALONÇO et al., 2006; BORTOLAIA; ANDRIGHETTO; BENATTI, 2008).

Com a realização deste trabalho objetivou-se desenvolver um protótipo revolvedor mecânico de grãos ou frutos de café semimecanizadopara o secador de camada estacionária visando substituir o revolvimento manual, com enxadas, aumentar a eficiência do processo de secagem, de revolvimento e, principalmente, minimizar o esforço do operador.

O revolvedor de café foi projetado e construído no Laboratório de Mecanização Agrícola (LMA) pertencente ao Departamento de Engenharia Agrícola da Universidade Federal de Viçosa (DEAUFV).Para o seu desenvolvimento, o projeto baseou-se nas metodologias propostas por Pahl et al. (2005), que consistem,basicamente,em:a valiar a inter-relação entre função, princípio de funcionamento e constituição;realizar buscas sobre o "estado da tecnologia" em exposições, boletins, catálogos, patentes, entre outros;realizar análise dos sistemas técnicos conhecidos através da análise de produtos concorrente e obsoletos similares.

Foram definidas as funções e características 
possíveis da máquina, sendo estas: revolver, transportar, movimentar a massa de grãos, quebrar a frente de secagem, descompactar camadas de grãos e minimizar o esforço do operador.Para a execução das tarefas citadas, uma equipe de engenheiros e técnicos realizou uma análise detalhada do problema e debateram idéias para suas possíveis soluções.Esta metodologia, descrita por Suares, Jung e Caten (2009), foi um misto entre os métodos de "brainstorming" e "brainwriting635".

Foram propostas soluções baseadas nos sistemas atuais de transporte de grãos, tais como: sistemas pneumáticos, transportadores helicoidais, correntes, correias transportadoras e elevadores de caçamba. Pontos importantes foram avaliados nas etapas de projeto e construção, tais como: o teor de água, maturação dos grãos de café, impurezas, altura da massa de grãos dentro da câmara de secagem, danos mecânicos, capacidade operacional e a demanda de potência para realizar o transporte da massa de grãos.Após compreensão e análise das propostas resolveu-se testar duas soluções exequíveis: as correias e os transportadores helicoidais.

O primeiro protótipo (Figura 1a) tinha como princípio básico transportar a massa de grãos de café, utilizando-se uma correia dotada de paletas de borracha. Para a continuidade do projeto deste primeiro protótipo foi utilizado a metodologia do Processo Geral de Decisão proposta por Phal et al. (2005) (Figura 1b) que foi utilizado concomitante aos ensaios para avaliar o princípio de revolvimento proposto para o segundo protótipo e a construção da capa externa apresentada para o terceiro protótipo. Após essa etapa, partiu-se para a execução do projeto, seleção dos materiais a serem utilizados na construção e para a realização dos ensaios verificando as interações entre os materiais do revolvedor e os grãos durante a operação de revolvimento do café.

As respostas positivas e negativas dentro do Processo Geral de Decisão (PHAL et al. 2005) (Figura 1b),demonstram se os resultados dos ensaios, em relação aos objetivos, foram ou não foram satisfatórios, permitindo que a equipe responsável pelo projeto seguisse para a etapa subsequente de ensaios das grandezas físicas já definidas de acordo com o plano de trabalho.O segundo(Figura 1c) e o terceiro protótipo (Figura 1d) foram projetados utilizando o princípio de transporte helicoidal, muito utilizado em transporte de grãos em unidades armazenadoras, sendo a inovação apresentada no terceiro protótipo, a construção de uma capa externa cilíndrica.

O fluxograma (Figura 1e) proposto por Phal et al. (2005), é um processo interativo, uma vez que algumas de suas etapas de trabalho precisam ser repetidas em um nível elevado de informação para que sirvam como base para as outras fases do projeto.

Para aperfeiçoamento do protótipo foi necessário dimensionar e construir um novo helicoide o mais leve possível e que atendesse às dimensões da câmara de secagem do secador de camada estacionária (Figura 1f). Para a realização dos ensaios dividiu-se a câmara de secagem em três partes com as mesmas medidas de largura, comprimento e altura, utilizadas como repetições dos ensaios. A restrição lateral em secadores de camada estacionária possibilita minimizar a área utilizada como câmara de secagem dos grãos, por outro lado necessita de revolvimento intensificado. 
Figura 1. (a) Croqui em CAD do primeiro protótipo revolvedor de grãos, (b) do diagrama do Processo geral de Decisão (PHAL et al., 2005), croquis do (c) terceiro e (d) do protótipo revolvedor.(e) Fluxograma das etapas de planejamento e (e) Secador de camada estacionária com restrição lateral da câmara de secagem.(a) CAD sketch of the first prototype of grains homogenizer (b) diagram of the general Decision Process, (c) Sketch of the second and (d) third homogenizer prototype. (e)Flowchart of the planning stages and (f)Dryer stationary layer with lateral restriction of drying chamber.
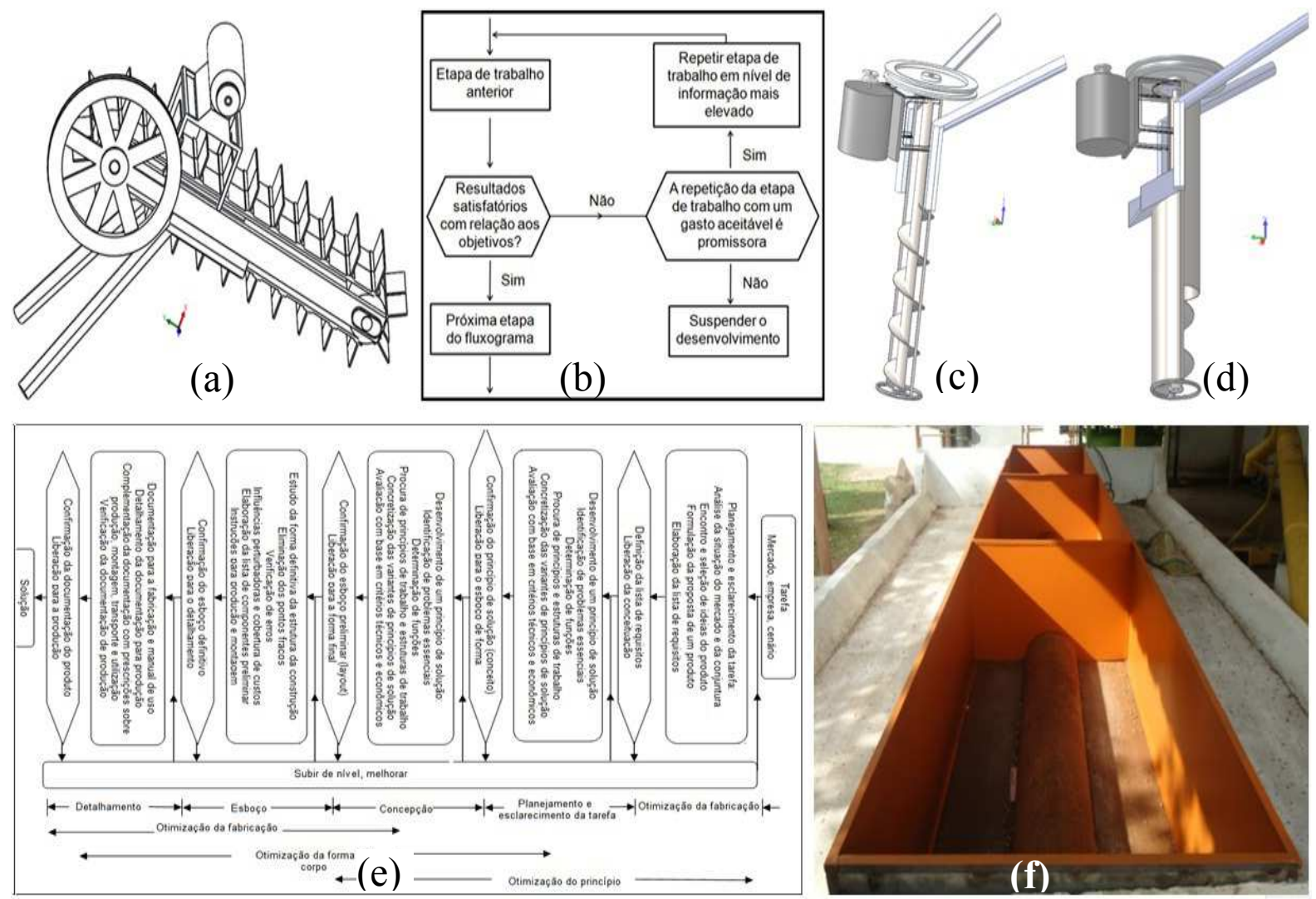

Fonte: (a), (c), (d) e (f), (b) e (e) Phal et al. (2005).

Para dimensionar o protótipo revolvedor foi necessário estimar as variáveis que afetam o desempenho de um helicoide (SRIVASTAVA et al., 2006). Estas foram calculadas em função das características dos grãos transportados e das condições de operação.

Para efeito de cálculos foram considerados os teores máximos e mínimos de água dos grãos de café. Inicialmente foram definidos o diâmetro externo do helicoide de $130 \mathrm{~mm}$, o passo de $130 \mathrm{~mm}$ e o menor diâmetro do eixo que fosse capaz de suportar soldas, de tal modo que o protótipo fosse o mais leve possível. Por esses motivos foi utilizado como eixo do helicoide um duto metálico com diâmetro e parede de $38,1 \mathrm{~mm}$ e $1,21 \mathrm{~mm}$, respectivamente.
Para o projeto do duto de transporte utilizou-se uma folga de $10 \mathrm{~mm}$ entre o helicoide e a parede externa, para evitar a interrupção do movimento giratório do helicoide, quebra e esmagamento dos grãos.

Técnicas de funilaria e as Equações 1, 2, 3 e 4 foram utilizadas para realizar os cálculos, montagem em sistema CAD e, posteriormente, a construção do helicoide pelo método passo a passo.

$$
r=\frac{h C_{e}}{A B-C e} r=\frac{h C_{e}}{A B-C_{e}}
$$




$$
\begin{aligned}
& \mathrm{R}=\mathrm{r}+\mathrm{h} \mathrm{R}=\mathrm{r}+\mathrm{h} \\
& \mathrm{Ce}=\sqrt{\left(g^{2}+\mathrm{O}^{2}\right)} \mathrm{Ce}=\sqrt{\left(g^{2}+\mathrm{d}^{2}\right)} \\
& \mathrm{AB}=\sqrt{\left(g^{2}+\mathrm{OD}^{2}\right)} \mathrm{AB}=\sqrt{\left(g^{2}+\mathrm{OD}^{2}\right)}
\end{aligned}
$$

em que,

$\mathrm{r}=$ raio interno da circunferência, $\mathrm{mm}$;

$\mathrm{h}=$ diferença de raios, $\mathrm{mm}$;

$\mathrm{Ce}=$ perímetro interno do helicoide, $\mathrm{mm}$;

$\mathrm{AB}=$ perímetro externo do helicoide, $\mathrm{mm}$;

$\mathrm{R}=$ raio externo do helicoide, $\mathrm{mm}$;

$\mathrm{d}=$ diâmetro externo do eixo, $\mathrm{mm}$;

$\mathrm{D}=$ diâmetro externo do helicoide, $\mathrm{mm}$, e

$\mathrm{g}=$ passo do helicoide, $\mathrm{mm}$.

O helicoide foi projetado e posteriormente construído com o objetivo de movimentar o máximo de café evitando o embuchamento, danos mecânicos e elevada demanda de potência, já que o motor escolhido para acionar o sistema transportador foi de baixa potência, consumo e peso.

No teste preliminar o revolvedor foi colocado para operar com frutos de café por duas horas interruptas. Neste teste foram verificados possíveis desgastes entre peças, o comportamento dos componentes do revolvedor com a massa de café e a resistência do equipamento ao ser operado.

$\mathrm{O}$ primeiro protótipo revolvedor (Figura 2a) apresentou problemas de travamento do sistema de transporte, pois durante o revolvimento os grãos depositavam-se entre a correia e os roletes. Utilizando-se do fluxograma do Processo Geral de Decisão (Figura 1b), o primeiro resultado dos ensaios foi de uma resposta negativa (não) seguida de outra positiva ( $\operatorname{sim}$ ). O que obrigou a realização de uma nova tentativa com um nível mais completo de informação.

A nova tentativa compreendeu em isolar as correias e os rolamentos contra a passagem de grãos. Ao realizar novos ensaios e aplicando novamente o Processo Geral de Decisão para os resultados, foram dadas respostas negativas (não), demonstrando que o problema de travamento não foi superado. Assim o princípio de correias e paletas para realizar o revolvimento da massa de café teve o seu desenvolvimento suspenso.

Para os próximos protótipos foi utilizado o princípio de transporte helicoidal para movimentação do café, sendo que para o desenvolvimento do helicoide foram utilizadas as dimensões resultantes das Equações 1, 2, 3 e 4 e a construção em sistema CAD (Figura 2b). Depois de definidas as dimensões do helicoide, passo de $130 \mathrm{~mm}$ por diâmetro externo de $130 \mathrm{~mm}$, iniciou-se a construção do mesmo com chapas e tubos de aço.

Durante os ensaios com o segundo protótipo (Figura 2c) verificou-se que na parte traseira do protótipo a massa de grãos era novamente succionada pelo helicoide, provocando o refluxo dos grãos revolvidos. Devido à ineficiência de revolvimento durante os ensaios este segundo protótipo recebeu no Processo Geral de Decisão resposta negativa (não) e positiva ( $\operatorname{sim}$ ) no fluxograma (Figura 1b), levando o mesmo para o processo de aperfeiçoamento e para os próximos ensaios. 
Figura 2. (a) Primeiro protótipo revolvedor de grãos, (b) desenvolvimento de um helicoide, (c) segundo protótipo revolvedor de grãos, (d) terceiro protótipo em operação de revolvimento.Croquis dorevolvedor (e)vista lateral, (f) frontal em corte, mecanismos de (g) descarga e (h) alimentação e (i) Teste do funcionamento do revolvedor com grãos de café.(a)First prototype of grains homogenizer, (b) development of an helical screw, (c)second prototype of grains homogenizer, (d) third prototype in revolving operation.Sketch of homozenizer(e)lateral view, (f) front cut-away view, (g) discharge mechanisms and (h) alimentation and(e) working tests of the homogenizer with coffee grains.
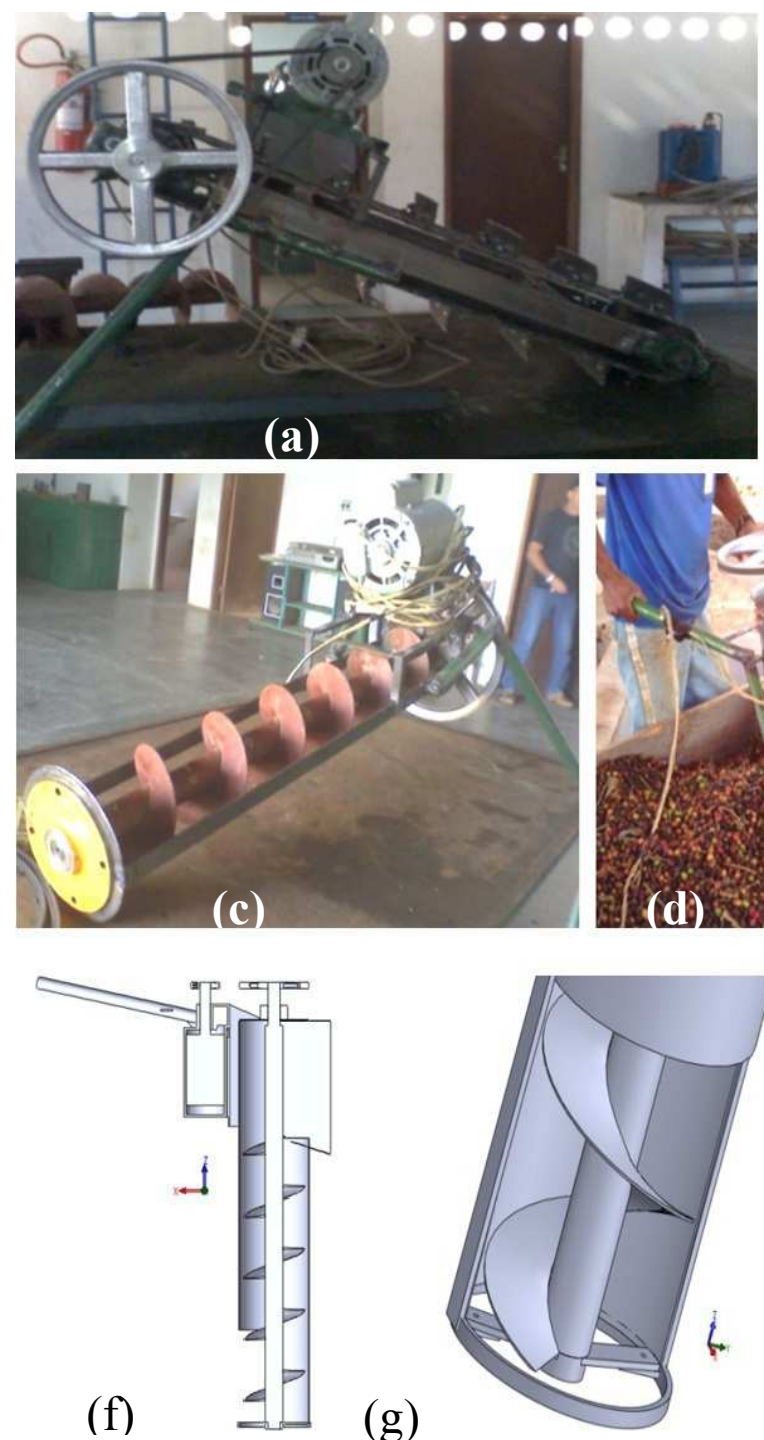

(g)

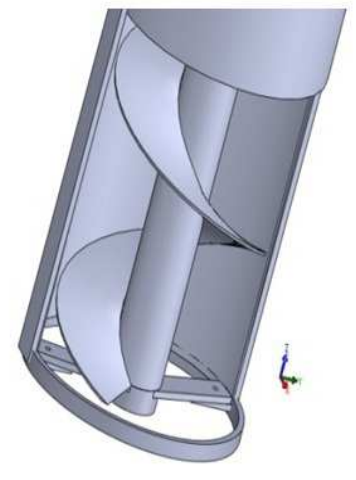

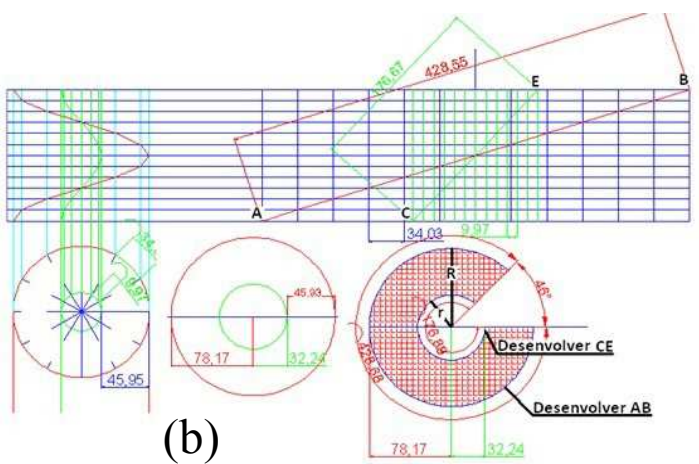
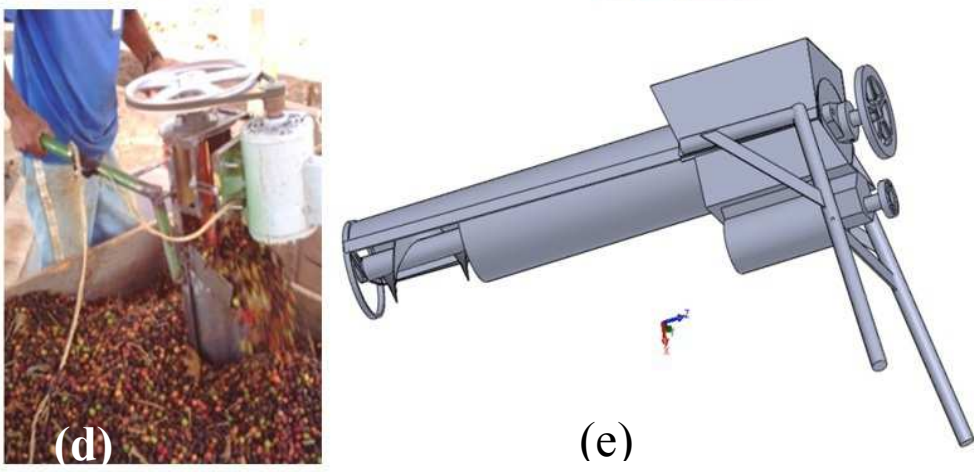

(e)
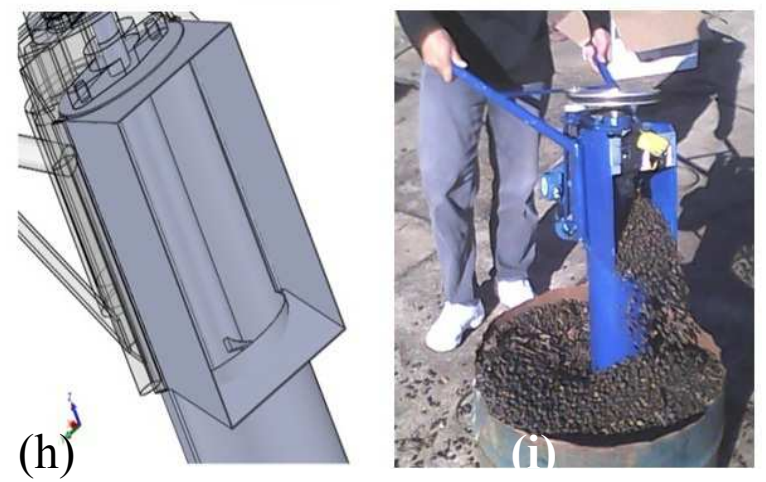

Fonte: Elaboração dos autores.

O terceiro protótipo (Figura 2d) foi resultado do aperfeiçoamento do protótipo anterior. Este teve a montagem de uma capa metálica externa ao helicoide para solucionar o problema de refluxo dos grãos. Durante os testes observou-se a movimentação da massa de grãos, mas ocorreram problemas, como peso excessivo do revolvedor, aproximadamente
$33 \mathrm{~kg}$, e da disposição do motor próximo à moega de descarga que dificultaram a movimentação do protótipo dentro da massa de grãos, acarretando problemas ergonômicos, pela elevada carga de trabalho imposta ao operador.

A fabricação de um novo protótipo com materiais mais leves e a instalação do motor entre as rabiças 
tornou o protótipo mais simples de ser operado. Para complementar as etapas de projeto do revolvedor, utilizou-se a proposta por Phal et al. (2005) (Figura 1e), onde foi confirmada a eficiência do princípio de transportador helicoidal dotada de duto.

No projeto do revolvedor (Figuras 1e e 1f) foram utilizadas chapas de aço de 1,21 $\mathrm{mm}$ para a montagem do tubo externo e da moega de descarga. Um mancal de rolamento tipo flange foi utilizado para fixar o eixo do helicoide no interior do tubo.

Tubos metálicos de diâmetro $38,1 \mathrm{~mm}$ foram usados como rabiças para realizar a condução do revolvedor.

O motor foi instalado sobre um chassi fixado na parte externa do revolvedor entre as rabiças. Um mancal tipo flange com bucha foi utilizado para a sustentação e centralização do helicoide na parte inferior. Para o acionamento do revolvedor utilizouse um motor de $250 \mathrm{~W}$ de potência. O sistema de acionamento foi constituído por uma polia motora, $150 \mathrm{~mm}$ de diâmetro e uma polia acionada, $50 \mathrm{~mm}$.

O sistema de alimentação (Figura 2g) dotado de uma abertura que proporcionou maior eficiência no transporte do café. A capacidade real de transporte é dependente das características dos frutos de café, das dimensões da moega de entrada, da rotação de trabalho e das dimensões do helicoide. Já o sistema de descarga (Figura $2 \mathrm{~h}$ ) foi projetado permitindo o transporte do café na direção oposta à alimentação, evitando assim o refluxo da massa de grãos de café.

Após a finalização do projeto do revolvedor em sistema CAD, a etapa seguinte, de acordo com a metodologia proposta por Phal et al. (2005) (Figura 1e), consistiu em confirmar o esboço, a forma final e iniciar a construção física do revolvedor em laboratório, seguindo as dimensões estabelecidas em projeto.

Seguida da construção do mesmo foram realizados testes preliminares em diversas rotações e com café em diferentes etapas de maturação, aproximando o funcionamento de uma situação real de operação (Figura 2i).
Nos testes preliminares foram verificadas falhas de construção das rabiças e da fixação da bucha. As rabiças não suportaram a movimentação do revolvedor, razão pela qual foram encontradas rachaduras nas soldas, que poderiam resultar em quebra e, por consequência, em acidentes. Prevenindo tal situação as soldas foram reforçadas e uma barra chata de 3 por 20 por $300 \mathrm{~mm}$ foi soldada como reforço da estrutura.Os parafusos de fixação da bucha, de suporte e centralizadora, apresentaram contato com a hélice do helicoide causando desgaste dos mesmos, quebra e esmagamento dos frutos. A solução foi a substituição por parafusos menores e de cabeça arredondada.

Depois de realizadas as modificações os testes foram repetidos, sendo verificada a solução dos problemas detectados anteriormente.

Entre os protótipos projetados, construídos e avaliados, somente o que utilizou o princípio de transporte helicoidal, trabalhando dentro de um tubo, realizou a movimentação da massa de café com maior eficiência esem apresentar embuchamentoou destruição dos grãos de café.

Com o fim dos ensaios foi possível verificar a grande possibilidade de ser utilizado o revolvedor mecânico para café como uma alternativa para o árduo trabalho de revolver e misturar a massa de grãos dentro de um secador de camada estacionária com restrição lateral da câmara de secagem.

O presente trabalho motivou a confecção e depósito do pedido de patente de modelo de utilidadeda máquina revolvedora de café, que se encontradisponível e protegido pelo Instituto Nacional de Propriedade Intelectual (INPI) sob o número MU9002333.

\section{Referências}

ALONÇO, A. dos S.; MEDEIROS, C. A.; MEDEIROS, F. A.;WERNER, V. Análise ergonômica do trabalho em atividades desenvolvidascom uma roçadora manual motorizada. Ciência Rural, Santa Maria, v. 36, n. 5, p. 1638-1642, set./out. 2006. 
AMPESSAN, F.; LACERDA FILHO, A. F. de; VOLK, M. B. da S.; RIGUEIRA, R. J. de A. Comparação entre secagens de café cereja descascado em terreiros com diferentes tipos de pavimentação. Engenharia na Agricultura, Viçosa, MG, v. 18, n. 5, p. 373-381, 2010.

BORTOLAIA, L. A.; ANDRIGHETTO, P. L.; BENATTI, M. Avaliação técnica de um transportador pneumático de grãos por aspiração.Ciência Rural, Santa Maria, v. 38, n. 2, p. 526-529, mar./abr. 2008.

CHAGAS, A. M.; TEIXEIRA, M. M.; PRAT, M. H. Construção e avaliação de uma máquina recolhedora de café em terreiro. Ciências Técnicas Agropecuárias, Cuba, v. 15. n. 2, p. 13-20, 2006.

CORADI, P. C.; BOREM, F. M.; OLIVEIRA, J. A. Qualidade do café natural e despolpado após diferentes tipos de secagem e armazenamento. Revista Brasileira de Engenharia Agrícola e Ambiental, Campina Grande, v. 12 , n. 2, p. 181-188, 2008.

MAGALHÃES, A. C.; TEIXEIRA, M. M.; COUTO, S. M.; RESENDE, R. C. de. Modelagem de máquina pneumática recolhedora de frutos de café em terreiro usando análise por elementos finitos. Engenharia Agrícola. Jaboticabal, v. 26, n. 2, p. 483-492, 2006.

MINETTE, L. J.; SILVA, E. P.; SOUZA, A. P.; SILVA, K. R. Avaliação dos níveis de ruído, luz e calor em máquina de colheita florestal. Revista Brasileira de Engenharia Agrícola e Ambiental, Campina Grande, v. 11, n. 6, p. 664-667, 2007.

MINETTE, L. J.; SOUZA, A. P. de; SILVA, E. P. da; MEDEIROS, N. M. Postos de trabalho e perfil de operadores de máquinas decolheita florestal. Ceres, Viçosa, v. 55, n. 1, p. 66-73, 2008.

PAHL, G.; BEITZ, W.; FELDHUSEN, J.; GROTE, K. H. Projeto na engenharia: fundamentos do desenvolvimento eficaz de produtos, métodos e aplicações. 6. ed. São Paulo: Editora Edgard Blücher, 2005.
RIGUEIRA, R. J. de A.; LACERDA FILHO, A. F. de; SILVA, J. S.; CECON, P. R.; PALACIN, J. J. F. Caracterização da qualidade do café (coffea arábica 1.) submetido a diferentes condições de secagem e armazenagem. Engenharia na Agricultura, Viçosa, MG, v. 15, n. 2, p. 151-167, 2007.

SAATH, R.; BORÉM, F. M.; ALVES, E.; TAVEIRA, J. H. da S.; MEDICE, R.; CORADI, P. C. Microscopia eletrônica de varredura do endosperma de café (Coffeaarabica L.) durante o processo de secagem. Ciência Agrotécnica, Lavras, v. 34, n. 1, p. 196-203, jan./ fev. 2010.

SANTOS, P. M. dos; SCHLOSSER, J. F.; ROMANO, L. N.; ROZIN, D.; TURATTI, J. da C.; WITTER, M. Prioridades de requisitos para projeto de postos de operação de tratores quanto à ergonomia e segurança. Pesquisa Agropecuária Brasileira, Brasília, v. 43, n. 7, p. 869-877, jul. 2008.

SHIGLEY, J. E.; MISCHKE, C. R.; BUDYNAS, R. G. Mechanicalen gineering desing. 7. ed. New York: Editora Bookman. Inc., 2004. 953 p.

SILVA, G. B.; ROLIM, M. M.; PEDROSA, E. M. R.; BEBÉ, F. V.; SILVA, E. F. F. Efeito da aplicação de água residuária da lavagem dos frutos de café sobre as propriedades químicas do solo. Engenharia Agrícola. Jaboticabal, v. 31, n. 1, p. 158-166, jan./fev. 2011.

SRIVASTAVA, A. K.; GOERING, C. E.; ROHRBACH, R. P.; BUCKAMASTER, D. R. Engineering principles of agricultural machines. 2. ed. Editora ASABE, 2006. $588 \mathrm{p}$.

SUARES, T. M.; JUNG, C. F.; CATEN, C. S. Adaptação e aplicação de um método de desenvolvimento de produtos em uma microempresa de manufatura de produtos decorativos. Revista $P \& D$ em Engenharia de Produção, Itajubá, v. 7, n. 1, p. 37-63, 2009. 